\title{
COMPUTING STRUCTURED SINGULAR VALUES FOR STURM-LIOUVILLE PROBLEMS
}

\author{
MUTTI-UR REHMAN ${ }^{1, *}$, GHULAM ABBAS ${ }^{1}$ AND ARSHAD MEHMOOD ${ }^{2}$ \\ ${ }^{1}$ Department of Mathematics, Sukkur IBA University, Sukkur-Sindh 65200 Pakistan \\ ${ }^{2}$ Department of Mathematics, The University of Lahore, Gujrat campus, Gujrat-Punjab 50700 Pakistan \\ ${ }^{*}$ Corresponding author: mutti.rehman@iba-suk.edu.pk
}

\begin{abstract}
In this article we present numerical computation of pseudo-spectra and the bounds of Structured Singular Values (SSV) for a family of matrices obtained while considering matrix representation of SturmLiouville (S-L) problems with eigenparameter-dependent boundary conditions. The low rank ODE's based technique is used for the approximation of the bounds of SSV. The lower bounds of SSV discuss the instability analysis of linear system in system theory. The numerical experimentation show the comparison of bounds of SSV computed by low rank ODE'S technique with the well-known MATLAB routine mussv available in MATLAB Control Toolbox.
\end{abstract}

\section{INTRODUCTION}

The spectrum of a matrix Sturm-Liouville (S-L) problem was characterized by F.V.Atkinson in terms of the spectrum of a class of unitary matrices in his famous book titled Discrete and continuous boundary value problems. The Atkinson's team had developed a prototype FORTRAN code in order to numerically compute the spectrum of S-L problems [1]. The spectrum of a regular, self-adjoint S-L problem is not bounded and therefore as a result is infinite. The spectrum of a regular, self-adjoint S-L problem is bounded and finite when the coefficients of S-L problem satisfies the external imposed conditions. For a positive integer $n$, exactly $n$ eigenvalues are computed for a class of S-L problems [2]. The finite spectrum to S-L problem while

Received 2019-06-12; accepted 2019-07-17; published 2019-09-02.

2010 Mathematics Subject Classification. 15A18, 15A03, 80M50.

Key words and phrases. eigen values; singular values; singular vectors; low rank ODE's.

(C)2019 Authors retain the copyrights of their papers, and all open access articles are distributed under the terms of the Creative Commons Attribution License. 
considering the transmission conditions are studied in [3] which shows the equivalent matrix representation. The matrix representation of S-L problems for eigen parameter dependent boundary conditions are studied in $[4-6]$.

A S-L problem is one of the form

$$
-\left(p y^{\prime}\right)^{\prime}+q y=\lambda W y
$$

on an open interval $I=(a, b)$ with $-\infty<a<b<\infty$ having the eigen parameter dependent boundary conditions of the form

$$
A_{\eta} \Theta(a)+B_{\eta} \Theta(b)=0, \quad \Theta=\left(\begin{array}{ll}
y & p y^{\prime}
\end{array}\right)^{t}
$$

with

$$
A_{\eta}=\left[\begin{array}{cc}
\eta \alpha_{1}^{\prime}-\alpha_{1} & -\eta \alpha_{2}^{\prime}+\alpha_{2} \\
0 & 0
\end{array}\right], B_{\eta}=\left[\begin{array}{cc}
0 & 0 \\
\eta \beta_{1}^{\prime}+\beta_{1} & -\eta \beta_{2}^{\prime}-\beta_{2}
\end{array}\right]
$$

where $\alpha_{i}, \alpha_{i}^{\prime}, \beta_{i}, \beta_{i}^{\prime} \in \mathbb{R}, \forall i=1: 2$, such that

$$
\xi_{1}=\alpha_{1}^{\prime} \alpha_{2}-\alpha_{1} \alpha_{2}^{\prime} \neq 0, \quad \xi_{2}=\beta_{1}^{\prime} \beta_{2}-\beta_{1} \beta_{2}^{\prime} \neq 0
$$

The parameter $\eta$ is the spectral parameter while the coefficients satisfying the minimal conditions $r=$ $\frac{1}{p}, q, w \in L(I, \mathbb{C})$ where $L(I, \mathbb{C})$ is the complex valued function which are Lebesgue integrable on the interval $I$.

The S-L problem as considered above is said to be of Atkinson type if for $n>1$ there exists

$$
a=a_{0}<b_{0}<\cdots<a_{n}<b_{n}=b
$$

with $r=\frac{1}{p}=0$ on $\left[a_{k}, b_{k}\right], W_{k}=\int_{a_{k}}^{b_{k}} W d W \neq 0, \forall k=1: n$ and $q=W=0$ on $\left[b_{k-1}, a_{k}\right], r_{k}=\int_{a_{k}}^{b_{k}} r d r \neq$ $0, \forall k=1: n$.

The S-L problem with the eigen parameter dependent boundary conditions is equivalent to matrix eigenvalue problem of the form

$$
(P+Q) U=\eta W U
$$

where $U=\left[v_{0}, u_{0}, u_{1}, \ldots, u_{n}, v_{n+1}\right]$ is an eigenvector. This finally lead us to the following eigenvalue problem where we aim to approximate eigenvalues $\eta_{i}$, singular values $\sigma_{i}$ and structured singular values $\mu_{i}$ of the matrix $(P+Q) W^{-1}$ with

$$
(P+Q) U=\eta W U
$$

in turns this implies that

$$
\left((P+Q) W^{-1}-\eta I\right) U=0
$$

The Singular Value Decomposition (SVD) is one of the important tool in the modern days numerical analysis and specially in numerical linear algebra. The applications of SVD and it's basic theory are studied in [7]. The main idea of SVD is based upon the factorization of the given matrix $A \in \mathbb{R}^{m, n}$ with $m \geq n$. The 
SVD tool splits up matrix $A$ into further three matrices $U, \Sigma$ and $V^{t}$. The matrix $\Sigma$ is the one look like as a diagonal matrix and having the non-negative quantities $\sigma_{i}$ along it's main diagonal. The quantities $\sigma_{i}$ are known as the singular values of $A$. The singular values are the positive square roots of the spectrum of matrix $A^{t} A$ rather than $A$. The columns vectors of $U$ are known as the left singular vectors of $A$ while the orthonormal eigen vectors of $A A^{t}$. On the other hand, the column vectors of the orthogonal matrix $V$ acts as the right singular vectors of $A$ and orthonormal eigen vectors for $A^{t} A$. Both singular values and singular vectors are relatively insensitive to the perturbations across the elements of the matrix under consideration. These quantities are also insensitive to finite precision error [8]. The singular values are well-conditioned with respect to an accuracy [9].

Golub-kahan-Reinsch singular value decomposition algorithm [10] is the standard numerical algorithm used for the approximation of the singular values of a matrix. Hestenes algorithm [11] and Kogbetliantz algorithm [12] acts as the parallel algorithms for the computation of the singular values $\sigma_{i}$. The algorithm by Golub-kahan-Reinsch is computationally very efficient on the sequential machine but however it's not much attractive on the parallel processor [13].

The Structured Singular Values (SSV) is the generalization of the singular values of a square, rectangular matrix $A \in \mathbb{K}^{m, n}$ with $\mathbb{K}=\mathbb{R}, \mathbb{C}$. SSV was first introduced by J.C.Doyle [14]. The SSV tool widely used in control, system theory to investigate both stability and instability of feedback systems. For applications we refer [15]. Unfortunately, the computation of an exact value of SSV is not possible and appear as an NP-hard problem [17] which allows to develop numerical methods in order to approximate bounds of SSV. The lower bounds of SSV are approximated by using power method [16] while [18] is used to approximate it's upper bounds. The lower bounds of SSV provide sufficient information about the instability of closed loop system while upper bounds is used to study the stability of feedback system in linear control theory.

\subsection{Preliminaries.}

Definition 1.1. The spectrum of a square a complex valued matrix $M \in \mathbb{C}^{n, n}$ is defined as

$$
\Lambda(M)=\{\lambda \in \mathbb{C}:|(\lambda I-M)|=0\}
$$

Definition 1.2. The pseudospectrum of a complex matrix $M \in \mathbb{C}^{n, n}$ with a small positive real parameter $\epsilon>0$ is defined as

$$
\Lambda_{\epsilon}(M)=\left\{\lambda \in \mathbb{C}:\left|(\lambda I-M)^{-1}\right| \geq \frac{1}{\epsilon}\right\} .
$$

Definition 1.3. For a small positive parameter $\epsilon \geq 0$. A number $\lambda$ belongs to epsilon-pseudo-spectrum of an operator $A$, denoted by $\Lambda_{\epsilon}(A)$ and satisfies the following equivalent conditions

(i) $\lambda \in \Lambda(A+E)$ for some perturbation $E$ having $\|E\| \leq \epsilon$; 
(ii) $\exists u \in \mathbb{C}^{n, n}$ having $\|u\|=1$ such that $\|A u-\lambda u\| \leq \epsilon$;

(iii) $\lambda \in \rho(A)$ and $\left\|(\lambda I-A)^{-1}\right\| \geq \frac{1}{\epsilon}$ or $\lambda \in \Lambda(A)$ where $\rho(A)$ denotes the spectral radius of the matrix $A$.

Definition 1.4. The pseudospectrum of a complex matrix $M \in \mathbb{C}^{n, n}$ with a small positive real parameter $\epsilon>0$ is defined as

$$
\Lambda_{\epsilon}(M)=\left\{\lambda \in \mathbb{C}:\left|(\lambda I-M)^{-1}\right| \geq \frac{1}{\epsilon}\right\}
$$

Definition 1.5. Unstructured uncertainty $\mathbb{B}$ or stuctured uncertainty $\mathbb{B}$ is stable transfer matrix or structured stable transfer matrix having the form.

$$
\mathbb{B}=\left\{\operatorname{diag}\left(\delta_{i} I_{i} ; \Delta_{j}\right): \delta_{i} \in \mathbb{C}, \Delta_{j} \in \mathbb{C}^{m_{j}, m_{j}}, \forall i=1: S, j=1: F\right\}
$$

Definition 1.6. For a given $n$-dimensional square matrix $M \in \mathbb{C}^{n, n}$ and underlying perturbation set $\mathbb{B}$, the $\mu$-value is defined as

$$
\mu_{\mathbb{B}}(M)=\frac{1}{\min \left\{\|\Delta\|_{2}: \Delta \in \mathbb{B}, \operatorname{det}(I-M \Delta)=0\right\}} .
$$

unless no such $\Delta$ cause $(I-M \Delta)$ to be singular for which $\mu_{\mathbb{B}}(M)=0$.

1.2. Reformulation of $\mu$-values. In this section we reformulate the $\mu$-values on the basis of structured spectral value sets. The key idea for the reformulation of the structured singular values is to shift the largest eigenvalue of the matrix valued function $I-M \Delta(t)$ such that for $\lambda_{\max }=1$ the new eigenvalue $\eta=0$ as $\eta=1-\lambda_{\max }$ and it achieve the maximum value to be one when $\lambda_{\max }=0$. On the basis of this mathematical construction, the reformulation of structured singular values is given as below.

Definition 1.7. For a given $M \in \mathbb{C}^{n, n}$ and perturbation level $\epsilon>0$, the structured spectral value set is denoted by $\Lambda_{\epsilon}^{\mathbb{B}}(M)$ and is defined as

$$
\Lambda_{\epsilon}^{\mathbb{B}}(M)=\left\{\lambda \in \Lambda(\epsilon M \Delta), \Delta \in \mathbb{B},\|\Delta\|_{2} \leq 1\right\}
$$

where $\Lambda(\epsilon M \Delta)$ denotes the spectrum of the matrix valued function $(\epsilon M \Delta)$, and is simply a disk centered at origin 0 .

Definition 1.8. The structured epsilon spectral value set for a given $M \in \mathbb{C}^{n, n}$ and $\epsilon \geq 0$, is defined as

$$
\Sigma_{\epsilon}^{\mathbb{B}}(M)=\left\{\eta: 1-\lambda: \lambda \in \Lambda_{\epsilon}^{\mathbb{B}}(M)\right\}
$$

Definition 1.9. For a given $M \in \mathbb{C}^{n, n}$ and an underlying perturbation set $\mathbb{B}$ the $\mu$-value is defined as

$$
\mu_{\mathbb{B}}(M)=\frac{1}{\arg \min _{\epsilon>0}\left\{\max |\lambda|=1, \lambda \in \Lambda_{\epsilon}^{\mathbb{B}}(M)\right\}}
$$




\section{Pseudo-Spectrum}

In this section we present the pseudospectra for matrices under consideration to whom the goal is to approximate structured singular values. For this purpose we make use of the software package EigTool [19]. EigTool is routinely used for plotting unstructured pseudospectra of the matrices under consideration. In Figures 1-4, we show the computation of the pseudospectra of a different matrices as taken in examples 1-4. We also show the absolute values and the real part of the eigenvalues computed for each matrix. The spectrum of the eigenvalues in 3-dimensional space is also shown by making use of Eigtool.

Let $A$ be an n-dimensional matrix and let $\Lambda(A)$ denotes the set of all the eigenvalues of the matrix $A$. Let $\|A\|$ denotes the matrix-norm of the matrix $A$ induced by an inner product space $\langle\cdot, \cdot\rangle$. The computation of the pseudo-spectra of an operator is very straightforward but at the same its very costly too. The boundaries associated with the pseudo-spectrum are nothing but just the level curves of the resolvent corresponding to

operator $A$, that is, $\left\|(\lambda I-A)^{-1}\right\|$. The computations of the level curves involves the computation of the numerical values of $\lambda$ at the grid point in the complex planes and then to compute the desired contour plots. For the computation of the $\epsilon$-pseudo-spectrum, the computation of an admissible perturbation $E$ such that $\|E\|=\epsilon$ for the perturbed matrix $A+E$. For the computation of the $\epsilon$-pseudo-spectrum the determination of the sets $L_{\epsilon}$ and $U_{\epsilon}$ is essential such that $L_{\epsilon} \leq \Lambda_{\epsilon} \leq U_{\epsilon}$.

Here, $L_{\epsilon}(A)=\left\{\lambda \in \rho(A): b(\lambda) \geq \frac{1}{\epsilon}\right\} \cup \Lambda(A)$ acts as a lower bound of the $\epsilon$-pseudo-spectrum with $\epsilon \geq 0$. For an upper bounds of the pseudo-spectrum, $U_{\epsilon}(A)=\left\{\lambda \in \rho(A): B(\lambda) \geq\left\|(\lambda I-A)^{-1}\right\|\right\}$ for all $\lambda \in \rho(A)$. For a complete detail we refer [20] and the reference therein.

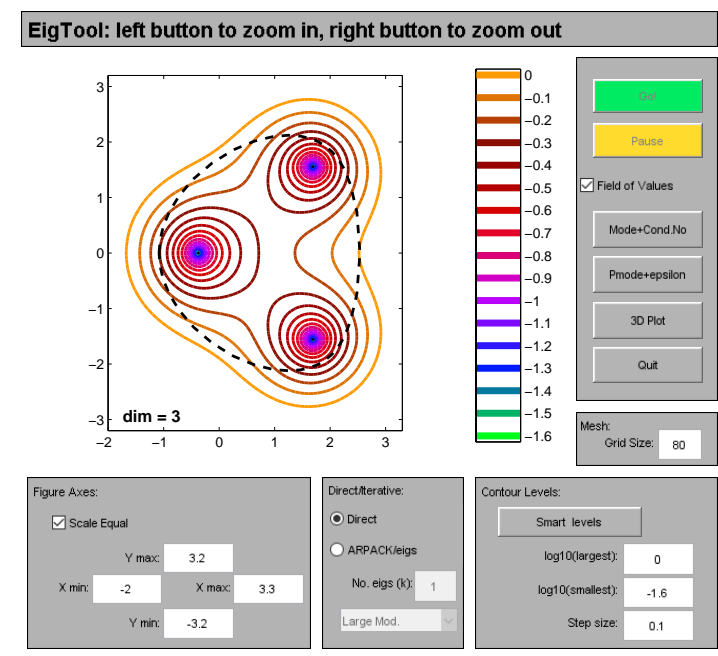

(A) Pseudospectrum of 3-dim real valued matrix

FiguRE 1. Matlab interface for computing pseudospectrum. The graphical representation show the pseudospectrum of the 3-dimensional real valued matrix (Example 1) 


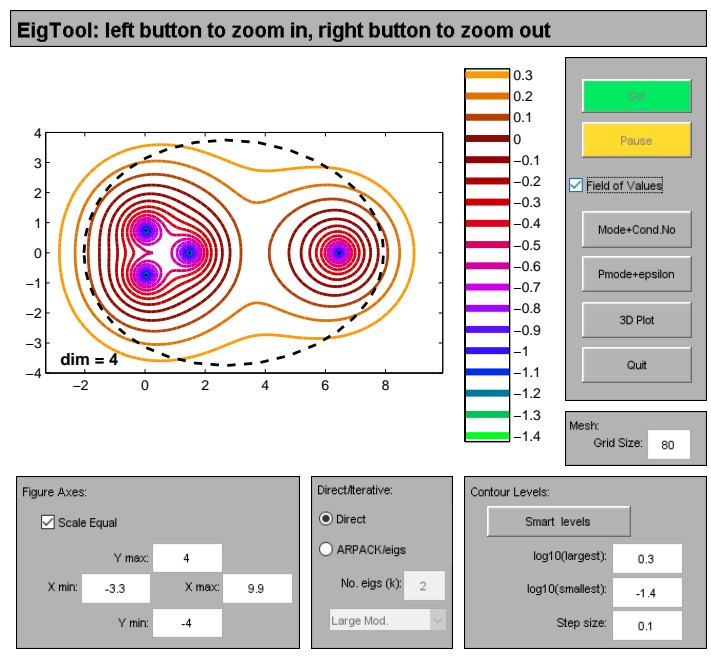

(A) Pseudospectrum of 4-dim real valued matrix

FIgURE 2. Matlab interface for computing pseudospectrum. The graphical representation show the pseudospectrum of the 4-dimensional real valued matrix (Example 2)

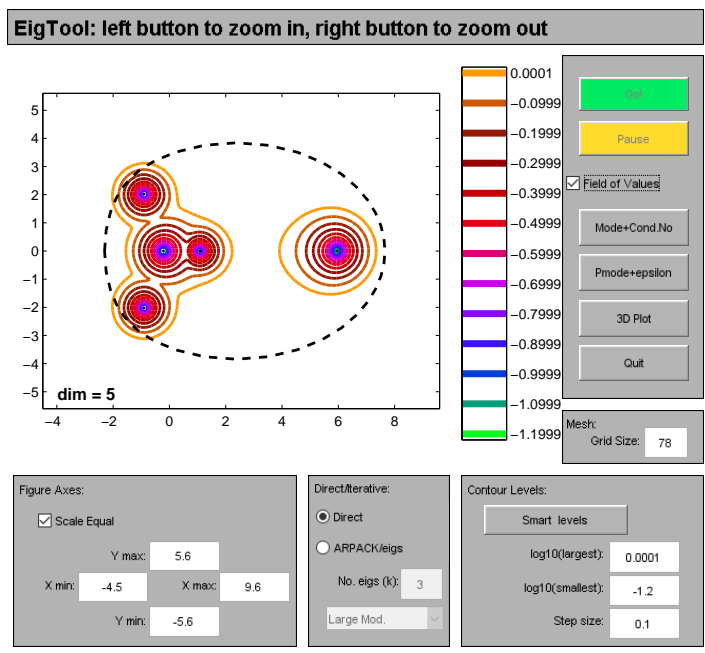

(A) Pseudospectrum of 5-dim real valued matrix

Figure 3. Matlab interface for computing pseudospectrum. The graphical representation show the pseudospectrum of the 5-dimensional real valued matrix (Example 3) 


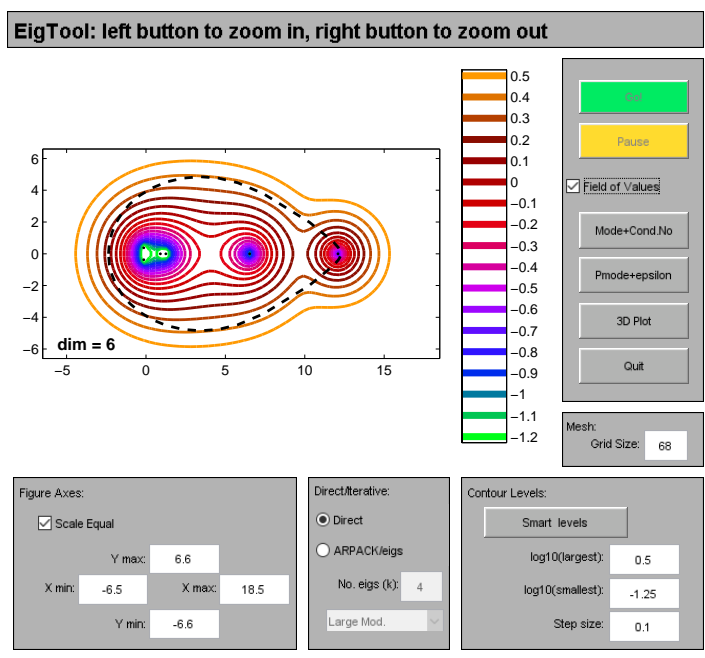

(A) Pseudospectrum of 6-dim real valued matrix

FiguRE 4. Matlab interface for computing pseudospectrum. The graphical representation show the pseudospectrum of the 6-dimensional real valued matrix (Example 4)

\section{Proposed Methodology}

In order to solve the maximization problem discussed in Definition 3.3, we make use of numerical method based upon low-rank ordinary differential equations technique. The numerical method is mainly composed of two-level algorithm, that is, inner-algorithm and outer-algorithm. In the inner-algorithm the main objective is to first construct then solve a gradient system of ordinary differential equations. On the other hand in the Outer-algorithm we vary the perturbation level $\epsilon>0$ by means of fast Newton iteration. The outeralgorithm computes an exact derivative of an extremizer say $\Delta(\epsilon)$ for $\Delta \in \mathbb{B}$ and $\epsilon>0$. A complete detail of numerical method under consideration is given in [15].

Next, we discuss the computation of an extremizer. For this purpose, we first approximate the derivative of an eigenvalue matrix $\Lambda(p)$ of a smooth matrix family say $A(p)$ for some fixed parameter $p$.

3.1. Approximation of an Extremizers. A matrix valued function $\Delta \in \mathbb{B}$ having the largest singular value bounded above by 1 and the matrix valued function $(I-\epsilon M \Delta)$ having a smallest eigenvalue which minimizes the modulus of structured spectral value set $\sum_{\epsilon}^{\mathbb{B}}(M)$ is known as an extremizer. The following theorem computes extremizer for a chosen smallest complex number belonging to the set $\sum_{\epsilon}^{\mathbb{B}}(M)$.

Theorem 5.1. For a perturbation $\Delta \in \mathbb{B}$ having the block diagonal structure

$$
\Delta=\left\{\operatorname{diag}\left(\delta_{1} I_{1}, \ldots \delta_{s^{\prime}} I_{s^{\prime}}, \delta_{s^{\prime}+1} I_{s^{\prime}+1}, \ldots \delta_{S} I_{S} ; \Delta_{1}, \ldots, \Delta_{F}\right\}\right.
$$


with $\|\Delta\|_{2}=1$, acts as a local extremizer of structured spectral value set. For a simple smallest eigenvalue $\lambda=|\lambda| e^{\iota \theta}, \theta \in \mathbb{R}$ of matrix valued function $(I-\epsilon M \Delta)$ having right and left evectors $x$ and $y$ scaled as $S=e^{\iota \theta} y^{*} x$ and let $z=M^{*} y$. The non-degeneracy conditions

$$
\begin{gathered}
z_{k}^{*} x_{k} \neq 0, \forall=1: S^{\prime} \\
\operatorname{Re}\left(z_{k}^{*} x_{k}\right) \neq 0, \forall=1: S^{\prime}+1: S \\
\text { and }\left\|z_{s+h}\right\| \cdot\left\|x_{s+h}\right\| \neq 0, \forall h=1: F,
\end{gathered}
$$

holds. Then magnitude of each complex scalar $\delta_{i} \forall i=1: s$ appears to be exactly equal to 1 while each full block possesses a unit 2-norm.

3.2. Gradiant System of ODE's. The gradient system of odes for an admissible perturbation $\Delta \in \mathbb{B}$ to approximate a local extremizer of smallest eigenvalue $\lambda=|\lambda| \epsilon^{i \theta}$, is obtained as,

$$
\begin{gathered}
\dot{\delta}_{i}=\nu_{i}\left(x_{i}^{*} z_{i}-\operatorname{Re}\left(x_{i}^{*} z_{i} \bar{\delta}_{i}\right) \delta_{i}\right) ; \quad i=1: s^{\prime} \\
\dot{\delta_{l}}=\operatorname{sign}\left(\operatorname{Re}\left(z_{l}^{*} x_{l}\right) \Psi_{(-1,1)}\left(\delta_{l}\right) ; \quad l=s^{\prime}+1: s\right. \\
\dot{\Delta_{j}}=\nu_{j}\left(z_{s+j} x_{s+j}^{*}-\operatorname{Re}\left\langle\Delta_{j} ; z_{s+j} x_{s+j}^{*}\right\rangle\right) ; \quad j=1: F,
\end{gathered}
$$

where $\delta_{i} \in \mathbb{C}, \forall i=1: s^{\prime}, \delta_{l} \in \mathbb{R}$ for $l=s^{\prime}+1$ and $\Psi_{(-1,1)}$, the characteristic function. For more discussion in the construction of gradient system of odes in above equations, we refer to [15].

3.3. Outer-Algorithm. In outer-algorithm the main aim is to vary $\epsilon>0$, the perturbation level by means of fast Newton's itaration. In turn $\frac{1}{\epsilon}$ will provide us the approximation of lower bound of $\mu$-value.

We make use of fast newton's iteration in order to solve a problem

$$
|\lambda(\epsilon)|=1
$$

In Eq. (5.1), $\epsilon>0$. In order to solve Eq. (5.1), we need to compute

$$
\frac{d}{d \epsilon}(|\lambda(\epsilon)|),
$$

the derivative.

The following theorem (5.2) help us to compute $\frac{d}{d \epsilon}(|\lambda(\epsilon)|)$, when $|\lambda(\epsilon)|$ is simple and $\Delta(0), \lambda(0)$ are assumed to remains smooth in the neighboring region of perturbation level $\epsilon>0$

Theorem 5.2 Consider matrix valued function $\Delta \in \mathbb{B}$. Let $x$ and $y$ as a function of perturbation level $\epsilon>0$ acts as right and left eigenvectors of matrix valued function $(\epsilon M \Delta)$. Consider the scaling of these vector accordingly of theorem (5.2). Let $z=M^{*} y$ and assume that non-degenracy conclusions as discussed in theorem (5.2) yields then,

$$
\begin{gathered}
\frac{d}{d \epsilon}(|\lambda(\epsilon)|)=\frac{1}{\left|y\left(\epsilon^{*}\right) x(\epsilon)\right|} \sum_{i=1}^{s}\left|z_{i}(\epsilon)^{*} x_{i}(\epsilon)\right| \\
+\sum_{j=1}^{F}\left\|z_{s+j}(\epsilon)\right\| \cdot\left\|y_{s+j}(\epsilon)\right\|>0 .
\end{gathered}
$$


3.4. Choice of suitable initial value matrix and initial perturbation level. For a suitable choice of the initial value matrix $\Delta_{0}$ and an initial perturbation level $\epsilon_{0}$, we refer to [15].

\section{Numerical Experimentation}

\section{Example 1.}

Consider a three dimensional real valued matrix $M=(P+Q) W^{-1}$ taken from [21].

$$
M=\left[\begin{array}{ccc}
-1 & -2 & 0 \\
1 & 2 & 2 \\
0 & -1 & 2
\end{array}\right]
$$

We take the underlying perturbation as

$$
\Theta_{\mathbb{B}}=\left\{\operatorname{diag}\left(\delta_{1} I_{1}, \Delta_{1}\right): \delta_{1} \in \mathbb{R}, \Delta_{1} \in \mathbb{C}^{2,2}\right\}
$$

The well-known MATLAB routine mussv approximates the bounds of SSV as follows along with the required perturbation $\widehat{\Delta}$ as

$$
\widehat{\Delta}=\left[\begin{array}{ccc}
-0.2877 & 0 & 0 \\
0 & 0.2124 & 0.0358 \\
0 & 0.1880 & 0.0316
\end{array}\right]
$$

We compute the matrix 2-norm of $\widehat{\Delta}$, that is, $\|\widehat{\Delta}\|_{2}=0.2877$. The mussv routine computes an upper bound $\mu_{P D}^{\text {upper }}=3.4762$ meanwhile a same lower bound is computed $\mu_{P D}^{\text {lower }}=3.4762$. Algorithm [15] computes the lower bounds of SSV as follows while the admissible perturbation $\epsilon^{*} \Delta^{*}$ is obtained as

$$
\Delta^{*}=\left[\begin{array}{ccc}
-1 & 0 & 0 \\
0 & 0.7384 & 0.1243 \\
0 & 0.6537 & 0.1100
\end{array}\right]
$$

The perturbation level is computed as $\epsilon^{*}=2$ and an admissible perturbation possesses a unit 2.norm, that is, $\left\|\Delta^{*}\right\|_{2}=1$. The lower bound of SSV is obtained as $\mu_{N e w}^{\text {lower }}=3.4762$.

Figure. 9 shows the numerical approximations of both lower and an upper bound of SSV. The graphical interpretation shows that in various cases the obtained results for the lower and upper bounds of SSV via Low rank ODE's and MATLAB routine mussv are similar. In some cases it's clear that obtained results via mussv for the lower bounds of SSV dominates than those obtained by Low rank ODE's technique. 


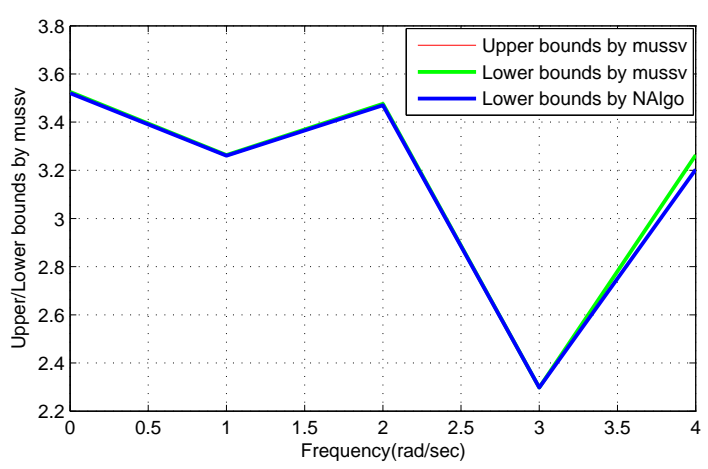

FIGURE 5. Comparison of the bounds of SSV approximated by MATLAB function mussv and NAlgo for a 3-dim real matrix valued function at frequencies $w=1: 5$.

Example 2. Consider a four dimensional real valued matrix $M=(P+Q) W^{-1}$ taken from [21].

$$
M=\left[\begin{array}{cccc}
-1 & -6 & 0 & 0 \\
1 & 6 & 1.3333 & 0 \\
0 & 4 & 2 & 1.5 \\
0 & 0 & 0.6667 & 1
\end{array}\right] .
$$

Figure. 10 shows the numerical approximations of both lower and an upper bound of SSV. The graphical interpretation shows that in various cases the obtained results for the lower and upper bounds of SSV via Low rank ODE's and MATLAB routine mussv are similar. In some cases it's clear that obtained results via mussv for the lower bounds of SSV dominates than those obtained by Low rank ODE's technique.

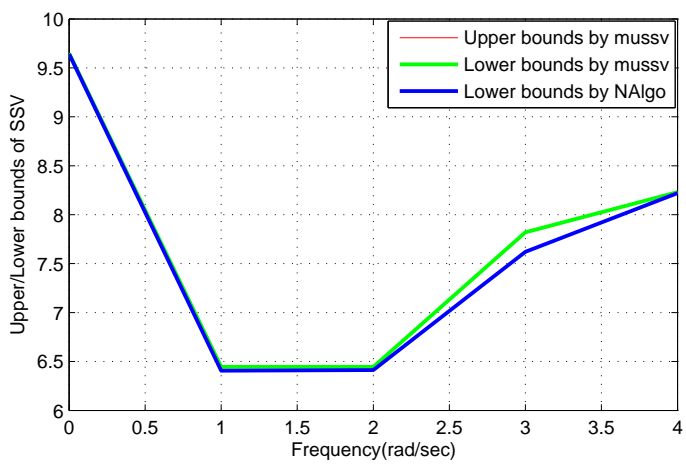

Figure 6. Comparison of the bounds of SSV approximated by MATLAB function mussv and NAlgo for a 4-dim real matrix valued function at frequencies $w=1: 5$. 


\section{Example 3.}

Consider a five dimensional real valued matrix $M=(P+Q) W^{-1}$ taken from [21].

$$
M=\left[\begin{array}{ccccc}
-1 & -6 & 0 & 0 & 0 \\
1 & 6 & 1.3333 & 0 & 0 \\
0 & 4 & 0 & -1.5 & 0 \\
0 & 0 & 2.6667 & -1 & 0.5 \\
0 & 0 & 0 & 0.5 & 1
\end{array}\right] .
$$

Figure. 11 shows the numerical approximations of both lower and an upper bound of SSV. The graphical interpretation shows that in various cases the obtained results for the lower and upper bounds of SSV via Low rank ODE's and MATLAB routine mussv are similar. In some cases it's clear that obtained results via mussv for the lower bounds of SSV dominates than those obtained by Low rank ODE's technique.

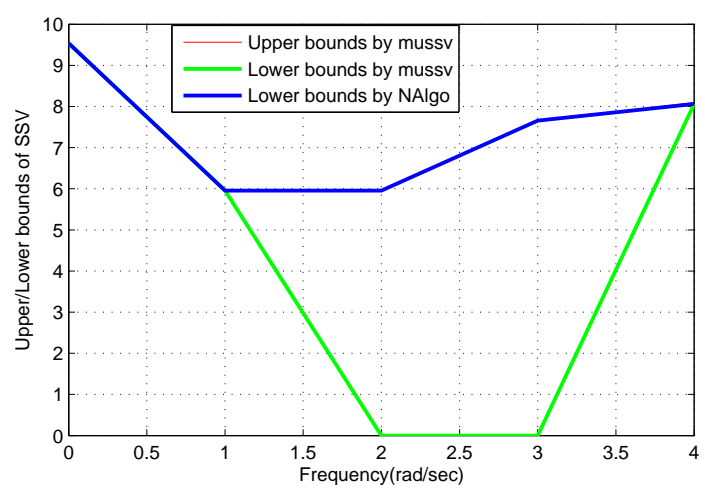

FiguRE 7. Comparison of the bounds of SSV approximated by MATLAB function mussv and NAlgo for a 5-dim real matrix valued function at frequencies $w=1: 5$.

\section{Example 4.}

Consider a six dimensional real valued matrix $M=(P+Q) W^{-1}$ taken from [21].

$$
M=\left[\begin{array}{cccccc}
-1 & -6 & 0 & 0 & 0 & 0 \\
1 & 6 & 1.3333 & 0 & 0 & 0 \\
0 & 4 & 0 & -1.5 & 0 & 0 \\
0 & -6 & -0.6667 & 2.5 & 0 & 0 \\
0 & 0 & 0 & -2 & 12 & 0.5 \\
0 & 0 & 0 & 0 & 2 & 1
\end{array}\right] .
$$

Figure. 12 shows the numerical approximations of both lower and an upper bound of SSV. The graphical interpretation shows that in various cases the obtained results for the lower and upper bounds of SSV via 
Low rank ODE's and MATLAB routine mussv are similar. In some cases it's clear that obtained results via mussv for the lower bounds of SSV dominates than those obtained by Low rank ODE's technique.

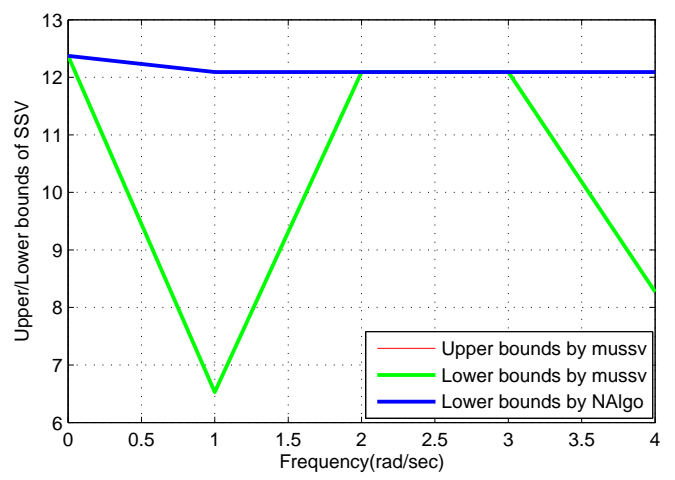

Figure 8. Comparison of the bounds of SSV approximated by MATLAB function mussv and NAlgo for a 6-dim real matrix valued function at frequencies $w=1: 5$.

\section{Conclusion.}

In this article we have presented numerical computation of pseudo-spectra and the bounds of structured singular values (SSV) for a family of matrices obtained while considering the matrix representation of Sturm-Liouville (S-L) problems with eigenparameter-dependent boundary conditions. The numerical experimentation shows that:

- In some cases the lower bounds of SSV obtained by Low rank ODE's based technique are sharper than the one approximated by MATLAB routine mussv.

- The MATLAB routine mussv is very fast compare to Low rank ODE's based technique.

- The MATLAB routine mussv additionally approximate an upper bounds of SSV which is not possible while making use of Low rank ODE's based technique.

\section{REFERENCES}

[1] Atkinson, FV and Krall, AM and Leaf, GK and Zettl, A. On the numerical computation of eigenvalues of matrix SturmLiouville problems with matrix coefficients. Argonne National Laboratory Reports, Darien, 1987.

[2] Kong, Q and Wu, H and Zettl, A. Sturm-Liouville problems with finite spectrum. J. Math. Anal. Appl., 263 (2001) $748-762$.

[3] Ao, Ji-jun and Sun, Jiong and Zhang, Mao-zhu. The finite spectrum of Sturm-Liouville problems with transmission conditions. Appl. Math. Comput., 218 (2001), 1166-1173.

[4] Kong, Qingkai and Volkmer, Hans and Zettl, Anton. Matrix representations of Sturm-Liouville problems with finite spectrum. Results Math., 54 (2009), 103-116. 
[5] Ao, Ji-jun and Sun, Jiong and Zhang, Mao-zhu. Matrix representations of Sturm-Liouville problems with transmission conditions. Computers Math. Appl., 63 (2012), 1335-1348.

[6] Ao, Ji-jun and Sun, Jiong and Zhang, Mao-zhu. The finite spectrum of Sturm-Liouville problems with transmission conditions and eigenparameter-dependent boundary conditions. Results Math., 63 (2013), 1057-1070.

[7] Vandewalle, Joos and De Moor, Bart. A variety of applications of singular value decomposition in identification and signal processing. SVD Signal Proc. Algorithms Appl. Architect Amsterdam, 1988, 43-91.

[8] Wilkinson, James Hardy. The algebraic eigenvalue problem. Oxford Clarendon, vol. 662, 1965.

[9] Klema, Virginia and Laub, Alan. The singular value decomposition: Its computation and some applications. IEEE Trans. Automatic Control, 25 (1980), 164-176.

[10] Golub, Gene and Kahan, William. Calculating the singular values and pseudo-inverse of a matrix. J. Soc. Ind. Appl. Math., Ser. B, Numer. Anal., 2 (1965), 205-224.

[11] Hestenes, Magnus R. Inversion of matrices by biorthogonalization and related results. J. Soc. Ind. Appl. Math., 6 (1958), $51-90$.

[12] Kogbetliantz, EG. Solution of linear equations by diagonalization of coefficients matrix. Q. Appl. Math., 13 (1955), $123-132$.

[13] Luk, Franklin T. Computing the singular value decomposition on the ILLIAC IV. Cornell University, year. 1980.

[14] Doyle, John. Analysis of feedback systems with structured uncertainties. IEE Proc., Part D , 129 (1982), 242-250.

[15] Guglielmi, Nicola and Rehman, Mutti-Ur and Kressner, Daniel. A novel iterative method to approximate structured singular values. SIAM J. Matrix Anal. Appl., 38 (2017), 361-386.

[16] Packard, Andy and Fan, Michael KH and Doyle, John. A power method for the structured singular value. Proc. 27th IEEE Conf. Decision Control, 1988, 2132-2137.

[17] Braatz, Richard P and Young, Peter M and Doyle, John C and Morari, Manfred. Computational complexity of $\mu$ calculation. IEEE Trans. Automatic Control 39 (1994), 1000-1002.

[18] Fan, Michael KH and Tits, André L and Doyle, John C. Robustness in the presence of mixed parametric uncertainty and unmodeled dynamics. IEEE Trans. Automatic Control 39 (1994), 25-38.

[19] Wright, Thomas G and Trefethen, LN. Eigtool. Software available at http://www.comlab.ox.ac.uk/pseudospectra/eigtool, 2002.

[20] Reddy, Satish C and Schmid, Peter J and Henningson, Dan S. Pseudospectra of the Orr-Sommerfeld operator. SIAM J. Appl. Math., 53 (1993), 15-47.

[21] Ao, Ji-jun and Sun, Jiong. Matrix representations of Sturm-Liouville problems with eigenparameter-dependent boundary conditions. Linear Algebra Appl., 438 (2013), 2359-2365. 